\title{
Possible mechanisms involved in the vasorelaxant effect produced by clobenzorex in aortic segments of rats
}

J. Lozano-Cuenca ${ }^{1}$, A. González-Hernández ${ }^{2}$, O.A. López-Canales ${ }^{3}$, J.R. Villagrana-Zesati ${ }^{4}$, J.D. Rodríguez-Choreño ${ }^{5}$, R. Morín-Zaragoza ${ }^{5}$, E.F. Castillo-Henkel ${ }^{3}$ and J.S. López-Canales ${ }^{1}$

${ }^{1}$ Department of Physiology and Cellular Development, National Institute of Perinatology, Mexico City, Mexico ${ }^{2}$ Department of Developmental Neurobiology and Neurophysiology, Institute of Neurobiology, National Autonomous University of Mexico, Queretaro, Mexico ${ }^{3}$ Section of Postgraduate Studies and Investigation, Higher School of Medicine, National Polytechnic Institute, Mexico City, Mexico ${ }^{4}$ Department of Infectology and Perinatal Immunology, National Institute of Perinatology, Mexico City, Mexico ${ }^{5}$ Mexican Academy for the Study of Obesity, Mexico City, Mexico

\begin{abstract}
Clobenzorex is a metabolic precursor of amphetamine indicated for the treatment of obesity. Amphetamines have been involved with cardiovascular side effects such as hypertension and pulmonary arterial hypertension. The aim of the present study was to investigate whether the direct application of $10^{-9}-10^{-5} \mathrm{M}$ clobenzorex on isolated phenylephrine-precontracted rat aortic rings produces vascular effects, and if so, what mechanisms may be involved. Clobenzorex produced an immediate concentrationdependent vasorelaxant effect at the higher concentrations $\left(10^{-7.5}-10^{-5} \mathrm{M}\right)$. The present outcome was not modified by $10^{-6} \mathrm{M}$ atropine (an antagonist of muscarinic acetylcholine receptors), $3.1 \times 10^{-7} \mathrm{M}$ glibenclamide (an ATP-sensitive $\mathrm{K}^{+}$channel blocker), $10^{-3} \mathrm{M}$ 4-aminopyridine (4-AP; a voltage-activated $\mathrm{K}^{+}$channel blocker), $10^{-5} \mathrm{M}$ indomethacin (a prostaglandin synthesis inhibitor), $10^{-5} \mathrm{M}$ clotrimazole (a cytochrome $\mathrm{P} 450$ inhibitor) or $10^{-5} \mathrm{M}$ cycloheximide (a general protein synthesis inhibitor). Contrarily, the clobenzorex-induced vasorelaxation was significantly attenuated $(P<0.05)$ by $10^{-5}$ M L-NAME (a direct inhibitor of nitric oxide synthase), $10^{-7} \mathrm{M} \mathrm{ODQ}$ (an inhibitor of nitric oxide-sensitive guanylyl cyclase), $10^{-6} \mathrm{M} \mathrm{KT} 5823$ (an inhibitor of protein kinase $\mathrm{G}$ ), $10^{-2} \mathrm{M}$ TEA (a Ca ${ }^{2+}$-activated $\mathrm{K}^{+}$channel blocker and non-specific voltage-activated $\mathrm{K}^{+}$ channel blocker) and $10^{-7} \mathrm{M}$ apamin plus $10^{-7} \mathrm{M}$ charybdotoxin (blockers of small- and large-conductance $\mathrm{Ca}^{2+}$-activated $\mathrm{K}^{+}$ channels, respectively), and was blocked by $8 \times 10^{-2} \mathrm{M}$ potassium (a high concentration) and removal of the vascular endothelium. These results suggest that the direct vasorelaxant effect by clobenzorex on phenylephrine-precontracted rat aortic rings involved stimulation of the NO/cGMP/PKG/Ca ${ }^{2+}$-activated $\mathrm{K}^{+}$channel pathway.
\end{abstract}

Key words: Clobenzorex; Rat aorta; Vasorelaxation; NO-cGMP; PKG pathway; $\mathrm{K}^{+}$channels

\section{Introduction}

Clobenzorex, $\mathrm{N}$-(2-clorobenzyl)-amphetamine is an appetite suppressant indicated for the treatment of obesity, available in many countries (e.g., Mexico) as a prescription drug. Its hepatic metabolism leads to the synthesis of: i) conjugated metabolites of clobenzorex or p-hydroxyclobenzorex; ii) amphetamine, p-hydroxyamphetamine or conjugated metabolites of $p$-hydroxyamphetamine, and iii) hippuric acid. Clobenzorex is one of several drugs metabolized to amphetamine and excreted in the urine $(1,2)$. Amphetamines used in weight management, such as sibutramine, fenfluramine and dexfenfluramine, have been involved with cardiovascular side effects such as hypertension and pulmonary arterial hypertension (3-5).

The cardiovascular side effects produced by amphetamines have been associated with an increased efflux of noradrenaline from the synaptic vesicles of sympathetic neurons, which interacts with vascular adrenoceptors such as $\alpha_{1}$-adrenoceptors to produce vasoconstriction $(6,7)$. However, there is evidence suggesting that the direct application of amphetamines on isolated rat aortic rings produces a vasoconstrictor effect through a mechanism not involving $\alpha_{1}$-adrenoceptors (8-10). On the other hand, it has been suggested that $N, N$-dimethyl-thioamphetamine, an amphetamine derivative, does not produce aortic contraction (11). Furthermore, recent evidence suggests that the direct application of fenproporex, a metabolic precursor to amphetamine, produces a vasorelaxant effect on phenylephrine precontracted aortic rings (12).

The present study aimed to analyze whether the direct application of clobenzorex, a metabolic precursor 
of amphetamine, produces vascular effects on rat aortic rings, and if so, what mechanisms may be involved.

\section{Material and Methods}

\section{Animals}

Experiments were performed on isolated thoracic aortic rings of adult male Wistar rats (weighing 250-300 g; $n=52$ ), purchased from the bioterium of the Higher School of Medicine of the National Polytechnic Institute (Mexico City). Animals were housed in plastic cages in a special temperature-controlled room $\left(22 \pm 2^{\circ} \mathrm{C}, 50 \%\right.$ humidity $)$ on a 12:12 $\mathrm{h}$ light/dark cycle (lights on at 7:00 am). The study was approved by the Animal Care Committee of the Higher School of Medicine and the protocol was in agreement with the 1986 Animals (Scientific Procedures) Act of the British Parliament: http://www.legislation.gov.uk/ ukpga/1986/14/contents (accessed on July 27, 2016).

\section{Preparation of aortic rings}

Animals were euthanized by decapitation and the aortas were immediately excised and placed in cold buffer, cleaned and freed from surrounding connective tissue. The isolated arteries were cut into rings (4-5 $\mathrm{mm}$ long) and placed in $10 \mathrm{~mL}$ tissue chambers filled with KrebsHenseleit bicarbonate buffer $\left(1.18 \times 10^{-1} \mathrm{M} \mathrm{NaCl} ; 4.7 \times\right.$ $10^{-3} \mathrm{M} \mathrm{KCl} ; 1.2 \times 10^{-3} \mathrm{M} \mathrm{KH}_{2} \mathrm{PO}_{4} ; 1.2 \times 10^{-3} \mathrm{M} \mathrm{MgSO}_{4}$. $7 \mathrm{H}_{2} \mathrm{O} ; 2.5 \times 10^{-3} \mathrm{M} \mathrm{CaCl}_{2} \cdot 2 \mathrm{H}_{2} \mathrm{O} ; 2.5 \times 10^{-2} \mathrm{M} \mathrm{NaHCO}_{3}$; $1.17 \times 10^{-2} \mathrm{M}$ dextrose, and $2.6 \times 10^{-5} \mathrm{M}$ calcium disodium EDTA). In some experiments, the concentration of $\mathrm{KCl}$ was increased to $8 \times 10^{-2} \mathrm{M}$ and that of $\mathrm{Na}^{+}$decreased to maintain osmotic equilibrium. Tissue baths, maintained at $37^{\circ} \mathrm{C}$ and $\mathrm{pH} 7.4$, were bubbled with a mixture of $95 \% \mathrm{O}_{2}$ and $5 \% \mathrm{CO}_{2}$.

Aortic rings were mounted on two stainless steel hooks, one fixed to the bottom of the chamber and the other to a BIOPAC TSD125C-50 g force transducer connected to a BIOPAC MP100A-CE data acquisition system (BIOPAC Systems, Inc., USA) in order to record the isometric tension. Optimal tension, selected from preliminary experiments, was that which gave the greatest response to $10^{-6} \mathrm{M}$ phenylephrine. The rings were given $2 \mathrm{~g}(100 \%)$ of initial tension and allowed to equilibrate for $2 \mathrm{~h}$. Thirty minutes after setting up the organ bath, tissues were contracted with $10^{-6} \mathrm{M}$ phenylephrine to test their contractile responses.

Endothelium-denuded aortic strips were prepared by turning the rings gently several times on the distal portion of small forceps. Endothelial integrity was pharmacologically assessed with acetylcholine-induced vasodilatation $\left(10^{-6} \mathrm{M}\right)$. Segments showing no relaxation to acetylcholine were considered to be endothelium-denuded. After exposure to $10^{-6} \mathrm{M}$ phenylephrine or $10^{-6} \mathrm{M}$ acetylcholine, tissues were rinsed three times with Krebs solution to restore basal tension.

\section{Drugs}

All drugs except clobenzorex were purchased from Sigma-Aldrich Co. (USA). Clobenzorex was a gift from Productos Medix, S.A. de C.V. Clobenzorex (Mexico), sodium nitroprusside (SNP), atropine, L-NAME, glibenclamide, 4-aminopyridine (4-AP), tetraethylammonium (TEA), clotrimazole and cycloheximide were dissolved in distilled

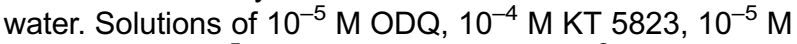
apamin plus $10^{-5} \mathrm{M}$ charybdotoxin and $10^{-3} \mathrm{M}$ indomethacin were prepared by using $1.39 \mathrm{M}$ dimethyl sulfoxide, $1.01 \mathrm{M}$ ethyl acetate, $1.73 \mathrm{M}$ acetic acid and $9.4 \times 10^{-3} \mathrm{M}$ sodium bicarbonate, respectively. Fresh solutions were made for each experiment.

\section{Experimental protocol}

To determine the mechanisms involved in the relaxant effect induced by clobenzorex on phenylephrine-precontracted rat aortic rings, two main sets of experiments were performed.

First set of experiments. Thirty minutes after restoration of basal tension, $10^{-6} \mathrm{M}$ phenylephrine was added to rat aortic rings with or without endothelium. Sixty minutes later, after phenylephrine-induced contraction plateaued, clobenzorex and SNP began to be cumulatively added $\left(10^{-9}-10^{-5} \mathrm{M}\right.$ and $10^{-11}-10^{-5} \mathrm{M}$, respectively) at intervals of around $5-6$ and $3-4 \mathrm{~min}$, respectively. Tension is reported as a percentage of the phenylephrine-induced contraction $(4.02 \pm 0.11 \mathrm{~g}=100 \%$ for endothelium-intact rat aortic rings and $4.39 \pm 0.17 \mathrm{~g}=100 \%$ for endotheliumdenuded rings).

Second set of experiments. Thirty minutes after adding $10^{-6} \mathrm{M}$ phenylephrine (see first set of experiments), aortic rings with intact endothelium were preincubated for 30 min with one (or two) of various compounds in order to explore the mechanisms involved in the vasorelaxant effect produced by clobenzorex. The compounds used for preincubation were: i) $10^{-6} \mathrm{M}$ atropine, a competitive muscarinic acetylcholine receptor antagonist; ii) $10^{-5} \mathrm{M}$ L-NAME, a direct inhibitor of NO synthase; iii) $10^{-7} \mathrm{M} O D Q$, an inhibitor of nitric oxide-sensitive guanylyl cyclase; iv) $10^{-6} \mathrm{M} \mathrm{KT} \mathrm{5823,} \mathrm{an} \mathrm{inhibitor} \mathrm{of} \mathrm{protein} \mathrm{kinase} \mathrm{G;}$ v) $3.1 \times 10^{-7} \mathrm{M}$ glibenclamide, an ATP-sensitive $\mathrm{K}^{+}$channel blocker; vi) $10^{-3} \mathrm{M}$ 4-aminopyridine (4-AP), a voltage-activated $\mathrm{K}^{+}$channel blocker; vii) $10^{-2} \mathrm{M}$ TEA, a Ca ${ }^{2+}$-activated $\mathrm{K}^{+}$channel blocker and nonspecific voltage-activated $\mathrm{K}^{+}$channel blocker; viii) $10^{-7} \mathrm{M}$ apamin plus $10^{-7} \mathrm{M}$ charybdotoxin, blockers of small- and large-conductance $\mathrm{Ca}^{2+}$-activated $\mathrm{K}^{+}$channels, respectively; ix) $10^{-5} \mathrm{M}$ indomethacin, a prostaglandin synthesis inhibitor; $x) 10^{-5} \mathrm{M}$ clotrimazole, a cytochrome P450 inhibitor; xi) $10^{-5} \mathrm{M}$ cycloheximide, a general protein synthesis inhibitor; xii) distilled water (vehicle of atropine, L-NAME, 4-AP, TEA, clotrimazole and cycloheximide), xiii) $1.39 \times 10^{-2} \mathrm{M}$ dimethyl sulfoxide (vehicle of ODQ), xiv) $1.01 \times 10^{-2} \mathrm{M}$ ethyl acetate (vehicle of KT 5823), xv) $1.73 \times 10^{-2} \mathrm{M}$ 
acetic acid (vehicle of apamin plus charybdotoxin), or $\mathrm{xvi}) 9.4 \times 10^{-5} \mathrm{M}$ sodium bicarbonate (vehicle of indomethacin). Subsequently, clobenzorex was cumulatively added $\left(10^{-9}-10^{-5} \mathrm{M}\right)$ at intervals of around $4 \mathrm{~min}$. Once reaching the desired concentration, the vasorelaxant response of the rings was assessed. In this way, the influence of the vehicles and drugs on the vasorelaxant response to $10^{-9}-10^{-5} \mathrm{M}$ clobenzorex was tested.

\section{Data analysis and statistics}

Data are reported as means \pm SE. In all experiments, $n$ equals the number of animals from which aortic segments were obtained (8 in each case). Values of maximal vasorelaxation $\left(E_{\max }\right)$ were analyzed by using the Student's $t$-test. Effects of inhibitors/blockers on the vasorelaxant responses produced by clobenzorex on phenylephrineprecontracted aortic segments were analyzed by using a two-way analysis of variance. Each analysis of variance was followed by a Student-Newman-Keul's post hoc test. Statistical significance was considered at $P<0.05$ (13). The statistical analysis was performed in the SigmaPlot 12 program (Systat Software Inc., USA).

\section{Results}

\section{Effect of clobenzorex on endothelium-intact and -denuded phenylephrine-precontracted rat aortic rings}

Figure 1A-D shows typical traces of the effect produced by the in vitro application of clobenzorex and SNP in endothelium-intact and endothelium-denuded phenylephrineprecontracted rat aortic rings. The addition of phenylephrine to endothelium-intact and endothelium-denuded rat aortic rings produced a sustained contraction. The cumulative addition of clobenzorex produced a concentration-dependent vasorelaxant response in endotheliumintact (Figure 1A), but not in endothelium-denuded (Figure 1B) phenylephrine-precontracted rat aortic rings. The cumulative addition of SNP produced a concentrationdependent vasorelaxant response in both endotheliumintact (Figure 1C) and endothelium-denuded (Figure 1D) phenylephrine-precontracted rat aortic rings. The $E_{\max }$ values presented a significant difference $(P<0.05)$ when comparing the effects of clobenzorex in endothelium-intact and -denuded phenylephrine-precontracted rat aortic rings: $110.07 \pm 2.69$ vs $5.49 \pm 0.82 \%$ for clobenzorex and $106.12 \pm 2.54$ vs $104.12 \pm 1.38 \%$ for SNP. $E_{50}$ values in endothelium-intact phenylephrine-precontracted rat aortic rings were $10^{-6.307} \mathrm{M}$ for clobenzorex and $10^{-7.436} \mathrm{M}$ for SNP.

\section{Effect of atropine on the vasorelaxation induced by clobenzorex in phenylephrine-precontracted rat aortic rings}

When comparing the effects of the absence and presence of atropine on the vasorelaxation induced by
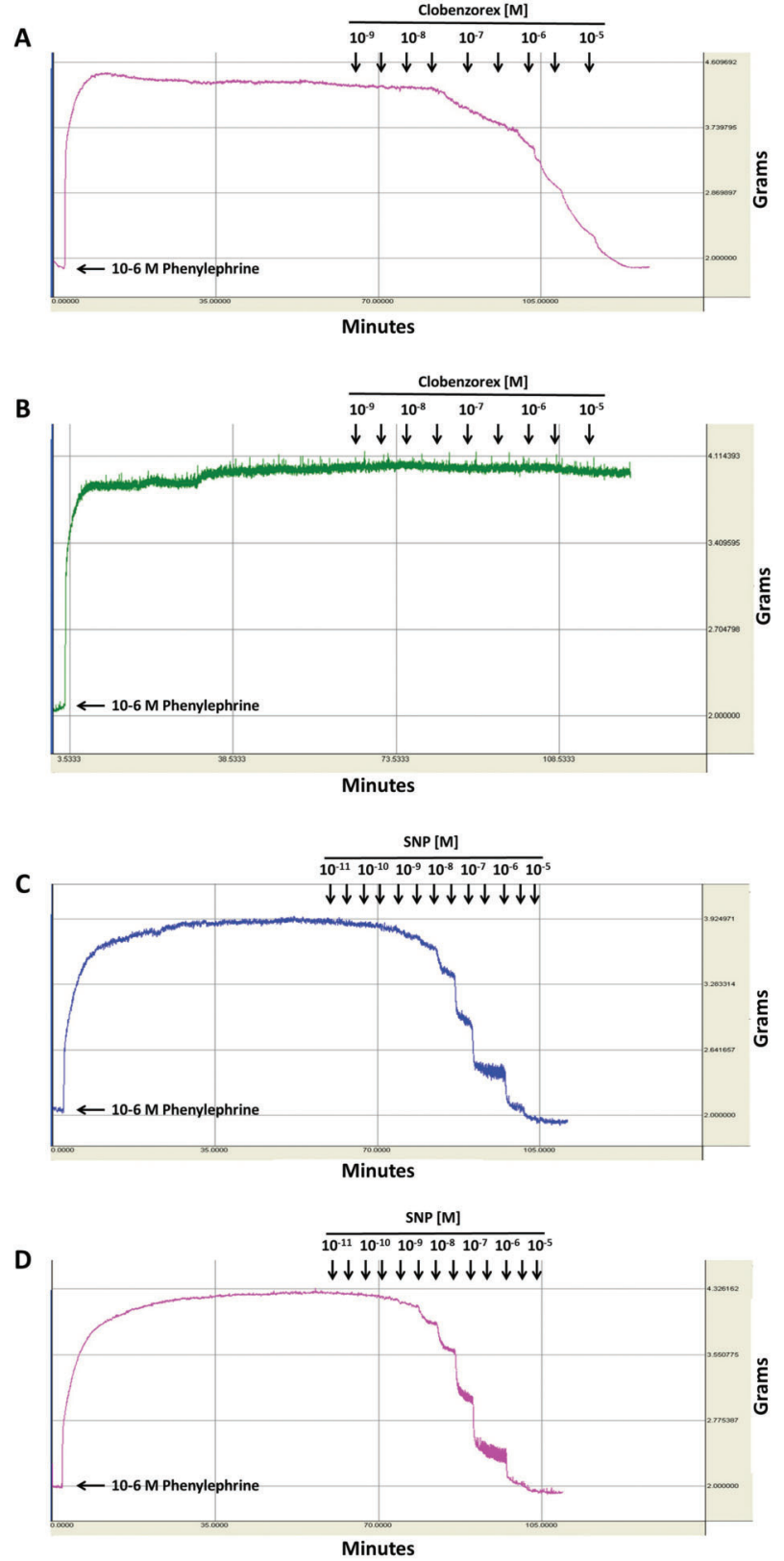

Figure 1. Representative tracings illustrating the relaxation response produced by the application of clobenzorex and sodium nitroprusside on endothelium-intact and endothelium-denuded phenylephrine-precontracted rat aortic rings. Clobenzorex $(A)$ and sodium nitroprusside (SNP) $(C)$ produced a dose-dependent vasorelaxant response in endothelium-intact phenylephrineprecontracted rat aortic rings. Endothelial denudation blocked the vasorelaxation to clobenzorex $(B)$, but not to SNP $(D)$. Similar results were obtained in all assays $(n=8)$.

clobenzorex in phenylephrine-precontracted rat aortic rings, the $E_{\max }$ values were not significantly different: $92.18 \pm 2.82$ vs $94.00 \pm 2.55$, respectively (Figure 2 ). 


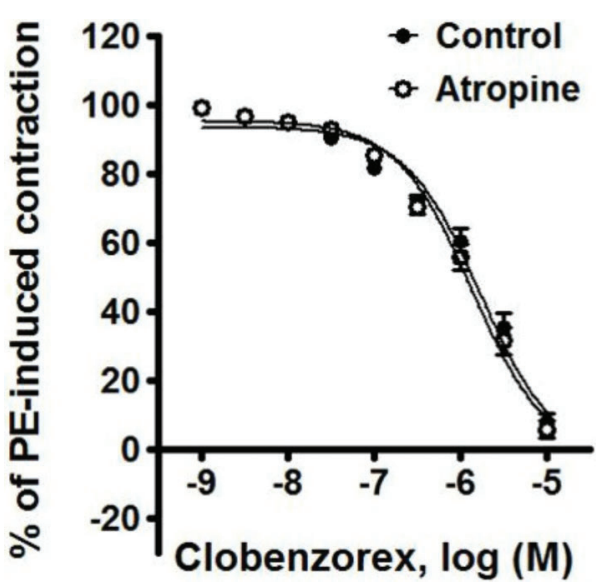

Figure 2. Pre-incubation with $10^{-6} \mathrm{M}$ atropine did not modify the vasorelaxation produced by $10^{-9}-10^{-5} \mathrm{M}$ clobenzorex in phenylephrine (PE)-precontracted rat aortic rings. Data are reported as means \pm SE of 8 observations.

Effect of L-NAME, ODQ and KT 5823 on the vasorelaxation induced by clobenzorex in phenylephrine-precontracted rat aortic rings

When comparing the effect of the absence and presence of L-NAME, ODQ, and KT 5823 on the vasorelaxation induced by clobenzorex in phenylephrine-precontracted rat aortic rings, the $E_{\text {max }}$ presented a significant difference $(P<0.05)$ in each case: $117.40 \pm 1.31$ vs $19.12 \pm 4.41 \%$ for L-NAME, $111.48 \pm 4.50$ vs $8.34 \pm 1.48 \%$ * for ODQ, and $95.77 \pm 2.94$ vs $10.82 \pm 2.42 \%$ for KT 5823 (Figure 3 ).

Effect of glibenclamide, 4-AP, TEA, and apamin plus charybdotoxin on the vasorelaxation induced by clobenzorex in phenylephrine-precontracted rat aortic rings

When comparing the effect of the absence and presence of glibenclamide, 4-AP, TEA, and apamin plus charybdotoxin on the vasorelaxation induced by clobenzorex in phenylephrine-precontracted rat aortic rings, the $E_{\max }$ presented a significant difference $(\mathrm{P}<0.05)$ only in the latter two cases: $116.19 \pm 1.63$ vs $108.26 \pm 4.24 \%$ for glibenclamide, $108.98 \pm 4.49$ vs $109.97 \pm 4.38 \%$ for 4 -AP, $113.28 \pm 2.74$ vs $19.07 \pm 2.80 \%$ for TEA, and $107.43 \pm$ 5.24 vs $6.49 \pm 1.22 \%$ for apamin plus charybdotoxin (Figure 4).

Effect of indomethacin, clotrimazole and cycloheximide on the vasorelaxation induced by clobenzorex in phenylephrine-precontracted rat aortic rings

When comparing the effect of the absence and presence of indomethacin, clotrimazole, and cycloheximide on the vasorelaxation induced by clobenzorex in phenylephrine-precontracted rat aortic rings, the $E_{\max }$ were not significant in any case: $103.16 \pm 3.52 \mathrm{vs}$
$100.23 \pm 5.29 \%$ for indomethacin, $118.18 \pm 2.45$ vs $117.36 \pm$ $2.45 \%$ for clotrimazole, and $102.94 \pm 5.50$ vs $100.88 \pm$ $4.32 \%$ for cycloheximide (Figure 5 ).

Effect of distilled water, dimethyl sulfoxide, ethyl acetate, acetic acid and sodium bicarbonate on the vasorelaxation induced by clobenzorex in phenylephrine-precontracted rat aortic rings

When comparing the effect of the absence and presence of the different vehicles on the vasorelaxation induced by clobenzorex in phenylephrine-precontracted rat aortic rings, the difference in the $E_{\max }$ was not significant in any case (Table 1).

\section{Discussion}

The acute application of clobenzorex produced an immediate concentration-dependent vasorelaxant effect on endothelium-intact but not on endothelium-denuded phenylephrine-precontracted rat aortic rings. The effect was statistically significant at higher concentrations $\left(10^{-7.5}\right.$ $10^{-5} \mathrm{M}$ ) of this appetite suppressant drug. The present results suggest that, by itself, clobenzorex, a metabolic precursor of amphetamine, produces an endotheliumdependent vasorelaxant effect. In this sense, the endothelium-dependent vasorelaxant effect of clobenzorex was reinforced by the results found with SNP (a nitric oxide donor drug). The latter compound, used as a positive control of endothelium-independent vasorelaxation, produced a concentration-dependent vasorelaxant effect on both endothelium-intact and -denuded phenylephrineprecontracted rat aortic rings, as previously reported $(14,15)$.

On the other hand, since the vehicle did not produce a concentration-dependent vasorelaxant effect in phenylephrine-precontracted rat aortic rings (data not shown), it can be ruled out that the clobenzorex-induced vasorelaxation was due to tachyphylactic effects caused by the repeated application of saline to aortic segments.

Clobenzorex produced a moderate vasodilator effect in the absence of phenylephrine-induced contraction. Nevertheless, we decided to perform our experimental protocol in rat aortic rings precontracted with phenylephrine to make evident the vasorelaxant effects produced by this appetite suppressant drug.

It is known that in the vasculature, the endothelial stimulation of muscarinic $M_{1}, M_{3}$, and $M_{5}$ receptors produces a vasorelaxant effect $(16,17)$. However, the fact that atropine, an antagonist of muscarinic acetylcholine receptors (18), did not modify the direct vasorelaxation produced by clobenzorex on rat aortic segments excludes the possible involvement of stimulation of muscarinic acetylcholine receptors in the vasodilator responses produced by this appetite suppressant drug.

The vasorelaxant effect produced by clobenzorex was significantly attenuated by L-NAME (a direct inhibitor of 

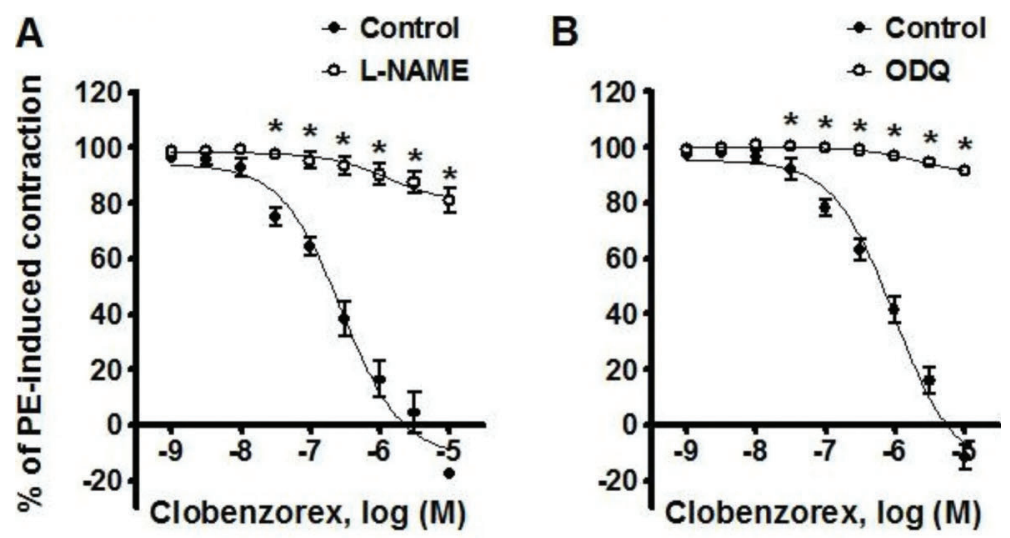

Figure 3. Vasorelaxation produced by $10^{-9}-10^{-5} \mathrm{M}$ clobenzorex in phenylephrine (PE)-precontracted rat aortic rings. Assays were carried out to test the effect of: $A, 10^{-5} \mathrm{M}$ L-NAME; $B, 10^{-7} \mathrm{M}$ ODQ; and $C, 10^{-6} \mathrm{M}$ KT 5823. Data are reported as means \pm SE of 8 observations. ${ }^{*} \mathrm{P}<0.05$ vs control (two-way ANOVA).

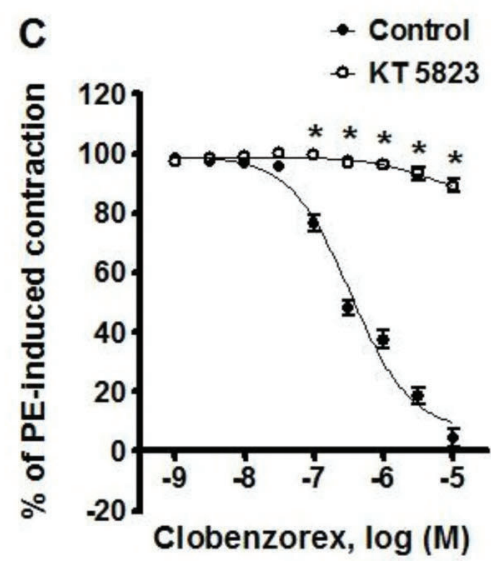

NOS) (19), ODQ (an inhibitor of nitric oxide-sensitive guanylyl cyclase) (20), and KT 5823 (an inhibitor of protein kinase G) (21). Hence, the stimulation of the NO-cGMP$P K G$ pathway is implied in the effect of this drug. In this sense, there is evidence that suggests that amphetamine can increase the NO synthesis in neurons of the striatal brain region through activation of NMDA receptors (22). This increase in the NO synthesis has been involved with stimulation of $M_{1}$ muscarinic acetylcholine receptors (23). However, the above evidence contrasts with the present study performed in rat aorta, in which, under the current experimental conditions, it was not possible to stimulate NMDA receptors. Moreover, since atropine did not modify the vasorelaxant effect produced by clobenzorex, it can be excluded the stimulation of muscarinic acetylcholine receptors. Indeed, we have no clear-cut explanation for the attenuating effect produced by L-NAME, ODQ and KT 5823. Possibly, clobenzorex enhances the activity or expression of the endothelial nitric oxide synthase producing the stimulation of the NO-cGMP-PKG pathway. However, this idea is still speculative and requires additional experiments that are beyond the scope of the present study.
On the other hand, the fact that the vasorelaxant effect produced by clobenzorex was unaffected by the respective vehicles of the L-NAME, ODQ and KT 5823 (distilled water, $1.39 \times 10^{-2} \mathrm{M}$ dimethyl sulfoxide and $1.01 \times 10^{-2} \mathrm{M}$ ethyl acetate) excludes the possibility that the attenuation of vasorelaxation produced by L-NAME, ODQ and KT 5823 was due to tachyphylactic effects induced by these vehicles.

The fact that the vasorelaxant effect produced by clobenzorex was unaffected by glibenclamide (an ATPsensitive $\mathrm{K}^{+}$channel blocker) (24) and 4-AP (a voltageactivated $\mathrm{K}^{+}$channel blocker) $(25,26)$, but significantly attenuated by TEA $\left(\mathrm{a} \mathrm{Ca}^{2+}\right.$-activated $\mathrm{K}^{+}$channel blocker and non-specific voltage-activated $\mathrm{K}^{+}$channel blocker) $(25,27)$ and apamin plus charybdotoxin (blockers of smalland large-conductance $\mathrm{Ca}^{2+}$-activated $\mathrm{K}^{+}$channels, respectively) (28-30) suggests the involvement of $\mathrm{Ca}^{2+}$ activated $\mathrm{K}^{+}$channels in the aforementioned effect. Furthermore, the vasorelaxant effect induced by clobenzorex was unaffected by distilled water (vehicle of L-NAME, 4-AP and TEA) and $1.73 \times 10^{-2} \mathrm{M}$ acetic acid (vehicle of apamin plus charybdotoxin). These results indicate that the vasorelaxation caused by clobenzorex is highly 

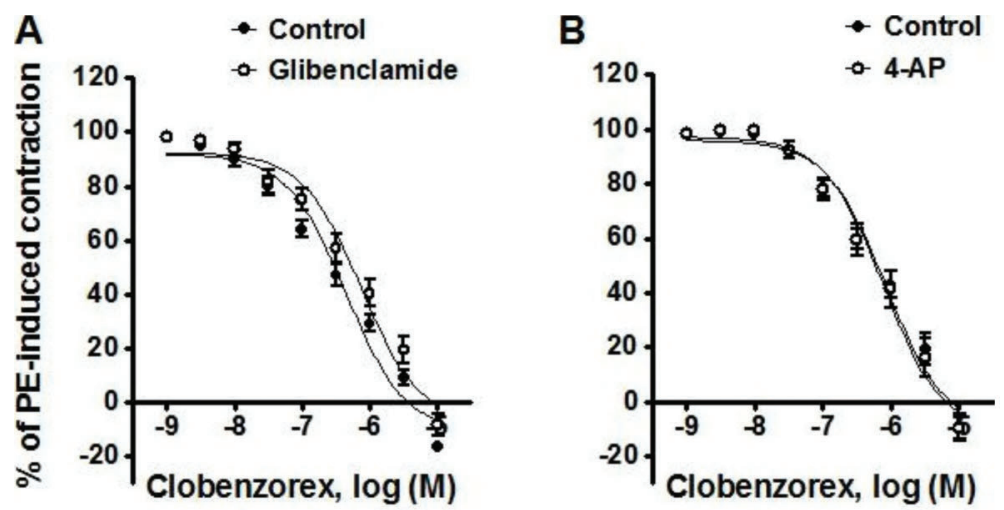

Figure 4. Vasorelaxation produced by $10^{-9}-10^{-5} \mathrm{M}$ clobenzorex in phenylephrine (PE)-precontracted rat aortic rings. Assays were carried out to test the effect of: $A, 3.1 \times 10^{-7} \mathrm{M}$ glibenclamide; $B, 10^{-3} \mathrm{M}$ 4-aminopyridine (4-AP); $C, 10^{-2} \mathrm{M}$ tetraethylammonium (TEA); and $D, 10^{-7} \mathrm{M}$ apamin (APA) plus $10^{-7}$ $M$ charybdotoxin (CHAR). Data are reported as means \pm SE of 8 observations. ${ }^{*} \mathrm{P}<0.05$ vs control (two-way ANOVA).
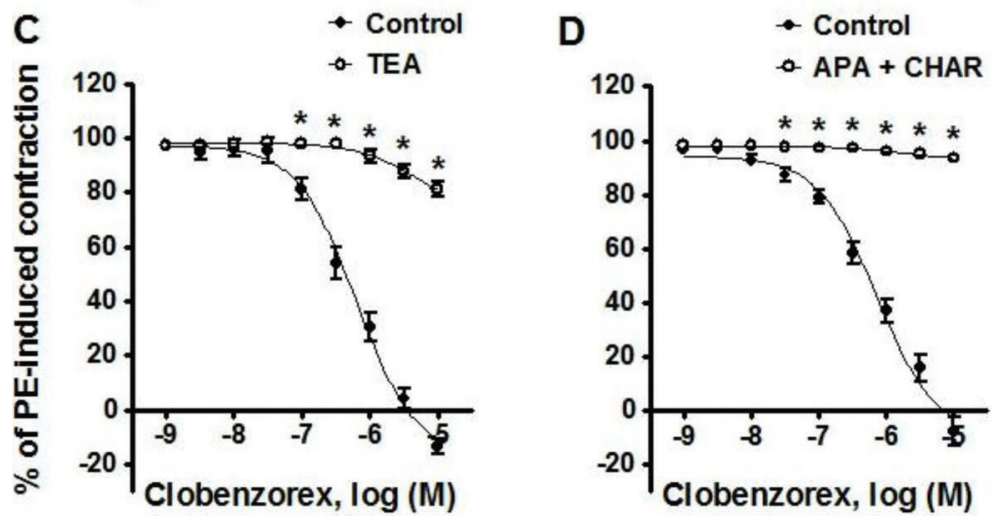

reproducible and rule out the possibility that attenuation produced by either of the $\mathrm{K}^{+}$channel blockers is due to tachyphylactic effects induced by their respective vehicles.

On the other hand, the combination of apamin plus charybdotoxin was used because it was previously reported that a complete blockage of $\mathrm{Ca}^{2+}$-activated $\mathrm{K}^{+}$ channels is necessary to produce a pharmacological response (29-31). In this sense, the two pilot experiments conducted in our laboratory showed that neither apamin nor charybdotoxin alone modified the vasorelaxant effect produced by clobenzorex (data not shown). Admittedly, we have no clear explanation about these observations, in which the combination of apamin plus charybdotoxin was necessary to block the vasorelaxant effect produced by clobenzorex. However, the experiments performed to elucidate whether the endothelium-derived hyperpolarizing factor EDHF could play a role in the vasorelaxant effect to clobenzorex, as previously reported for acetylcholine $(32,33)$, are discussed below.

It has been suggested that additional mechanisms are involved in the endothelial control of vascular tone, such as prostacyclins (34) and EDHF, a cytochrome P450derived arachidonic acid metabolite $(35,36)$. However, the fact that the vasorelaxation produced by clobenzorex was unaffected by indomethacin, a prostaglandin synthesis inhibitor (37), clotrimazole, a cytochrome P450 inhibitor (36) and cycloheximide, a general protein synthesis inhibitor (38) excludes the involvement of prostacyclins, EDHF and protein synthesis in the endothelium-mediated vasorelaxation under the current experimental conditions. In this sense, the lack of effect of clotrimazole supports previous studies that suggest that EDHF plays no role in the endothelium-dependent relaxation in the rat aorta (39). Moreover, it must be emphasized that the concentration of cycloheximide used presently was high enough to block the protein synthesis (31).

The present study shows that the direct and acute in vitro application of clobenzorex to rat aortic rings produces an endothelium-dependent vasorelaxant effect. However, the in vitro character of this study represents a limitation. Although the current findings suggest a direct vasorelaxant effect of clobenzorex, in vivo studies are needed to establish whether the systemic administration of this appetite suppressant drug produces a vasodepressor effect. Moreover, clobenzorex is clinically used in the chronic treatment of obesity. However, the experiments of the present study were performed to analyze the effect of the direct application of clobenzorex in aortic 

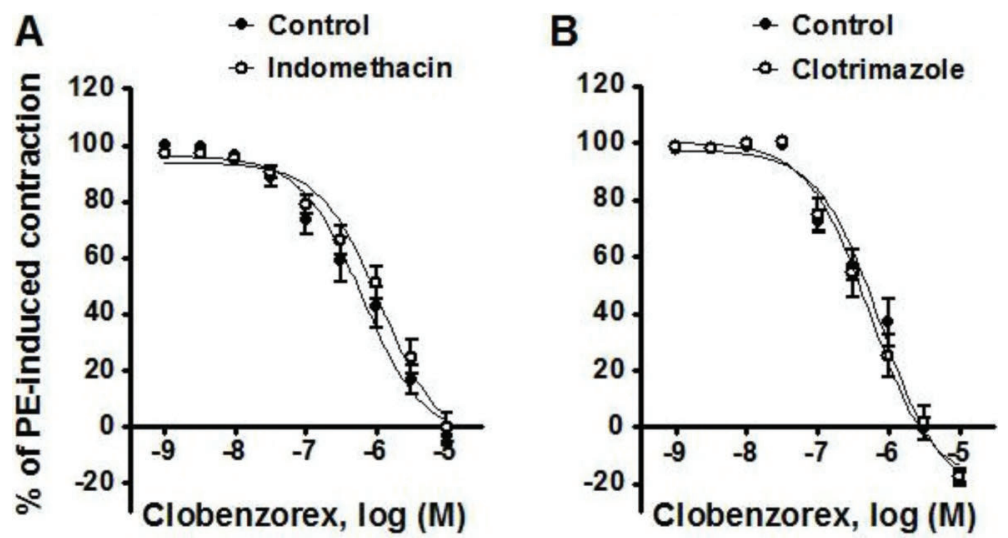

Figure 5. Vasorelaxation produced by $10^{-9}-10^{-5}$ M clobenzorex in phenylephrine-precontracted rat aortic rings. Assays were carried out to test the effect of: $A, 10^{-5} \mathrm{M}$ indomethacin; $B, 10^{-5} \mathrm{M}$ clotrimazole; and $C, 10^{-5} \mathrm{M}$ cycloheximide. Data are reported as means \pm SE of 8 observations.

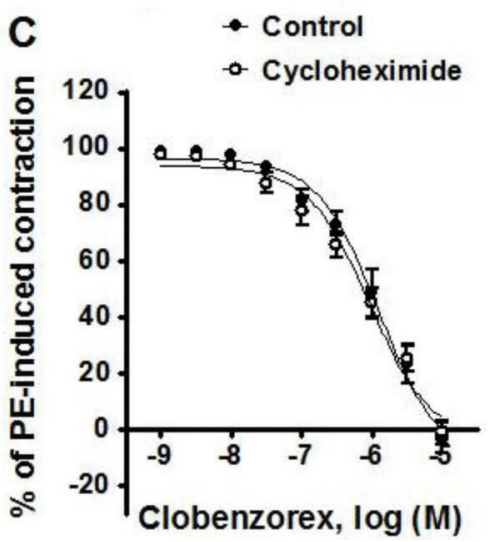

Table 1. Effect of the absence and presence of the vehicles on the maximal vasorelaxation induced by clobenzorex in phenylephrine-precontracted rat aortic rings.

\begin{tabular}{lrr}
\hline Vehicle & \multicolumn{2}{c}{ Maximal vasorelaxation to clobenzorex $\left(E_{\max }\right)$} \\
\cline { 2 - 3 } & \multicolumn{1}{c}{ Absence } & \multicolumn{1}{c}{ Presence } \\
\hline Distilled water & $99.55 \pm 1.39 \%$ & $99.97 \pm 1.68 \%$ \\
$1.39 \times 10^{-2} \mathrm{M}$ dimethyl sulfoxide & $100.91 \pm 2.48 \%$ & $101.78 \pm 5.66 \%$ \\
$1.01 \times 10^{-2} \mathrm{M}$ ethyl acetate & $98.81 \pm 3.25 \%$ & $96.72 \pm 3.01 \%$ \\
$1.73 \times 10^{-2} \mathrm{M}$ acetic acid & $112.12 \pm 2.89 \%$ & $113.52 \pm 1.17 \%$ \\
$9.4 \times 10^{-5} \mathrm{M}$ sodium bicarbonate & $109.37 \pm 5.62 \%$ & $111.86 \pm 3.24 \%$ \\
\hline
\end{tabular}

Data are reported as means \pm SE of 8 observations.

segments of rats with normal weight. Admittedly, further experiments are needed to analyze the vascular effects produced by clobenzorex in obese animals. Overall, the present results suggest that the NO/cGMP/PKG/Ca ${ }^{2+}$ activated $\mathrm{K}^{+}$channel pathway was a possible mechanism for the vasorelaxant effect observed.

\section{Acknowledgments}

The authors greatly appreciate the technical assistance of Lizeth Ledesma Rodea and Oscar Martín Boche Olivan (National Institute of Perinatology, Mexico City, Mexico). 


\section{References}

1. Valtier S, Cody JT. Metabolic production of amphetamine following administration of clobenzorex. J Forensic Sci 1999; 44: 17-22, doi: 10.1520/JFS14406J.

2. Glasson B, Benakis A, Thomasset M. (Localisation, distribution, excretion and metabolism of the new, C14-labelled appetite depressant clobenzorex hydrochloride). Arzneimittelforschung 1971; 21: 1985-1992.

3. Seferian A, Chaumais MC, Savale L, Gunther S, TubertBitter $\mathrm{P}$, Humbert $\mathrm{M}$, et al. Drugs induced pulmonary arterial hypertension. Presse Med 2013; 42 (Part 2) e303-e310, doi: 10.1016/j.Ipm.2013.07.005.

4. Franko BV, Honkomp LJ, Ward JW. Cardiovascular and autonomic effects of fenfluramine hydrochloride. J Pharm Pharmacol 1965; 17: 222-226, doi: 10.1111/j.2042-7158. 1965.tb07648.x.

5. Scheen AJ. Cardiovascular risk-benefit profile of sibutramine. Am J Cardiovasc Drugs 2010; 10: 321-334, doi: 10.2165/ 11584800-000000000-00000.

6. Schonfeld CL, Trendelenburg U. The release of $3 \mathrm{H}$-noradrenaline by $p$ - and $m$-tyramines and -octopamines, and the effect of deuterium substitution in alpha-position. Naunyn Schmiedebergs Arch Pharmacol 1989; 339: 433-440, doi: 10.1007/BF00736058.

7. Axelrod J, Whitby LG, Hertting G. Effect of psychotropic drugs on the uptake of $\mathrm{H} 3$-norepinephrine by tissues. Science 1961; 133: 383-384, doi: 10.1126/science.133.3450.383.

8. Broadley KJ, Fehler M, Ford WR, Kidd EJ. Functional evaluation of the receptors mediating vasoconstriction of rat aorta by trace amines and amphetamines. Eur J Pharmacol 2013; 715: 370-380, doi: 10.1016/j.ejphar.2013.04.034.

9. Baker KE, Herbert AA, Broadley KJ. Vasoconstriction of porcine left anterior descending coronary artery by ecstasy and cathinone is not an indirect sympathomimetic effect. Vascul Pharmacol 2007; 47: 10-17, doi: 10.1016/j.vph.2007.03.001.

10. Fehler M, Broadley KJ, Ford WR, Kidd EJ. Identification of trace-amine-associated receptors (TAAR) in the rat aorta and their role in vasoconstriction by beta-phenylethylamine. Naunyn Schmiedebergs Arch Pharmacol 2010; 382: 385398, doi: 10.1007/s00210-010-0554-1.

11. Sotomayor-Zarate R, Jara P, Araos P, Vinet R, Quiroz G, Renard GM, et al. Improving amphetamine therapeutic selectivity: N,N-dimethyl-MTA has dopaminergic effects and does not produce aortic contraction. Basic Clin Pharmacol Toxicol 2014; 114: 395-399, doi: 10.1111/bcpt.12168.

12. Lopez-Canales JS, Lozano-Cuenca J, Morin-Zaragoza R, Rodriguez-Almaraz JE, Lopez-Canales OA, Ruiz-Frutos C, et al. Posible mecanismo involucrado en el efecto vasodilatador inducido por fenproporex en anillos aorticos de rata. Revista del Hospital Juarez de Mexico 2013; 80: 235-242.

13. Steel RGD, Torrie JH. Principles and procedures of statistic: a biomedical approach. McGraw-Hill, New York; 1997.

14. Lockette $\mathrm{W}$, Otsuka $\mathrm{Y}$, Carretero $\mathrm{O}$. The loss of endotheliumdependent vascular relaxation in hypertension. Hypertension 1986; 8 (Part 2): II61-II66, doi: 10.1161/01.HYP.8. 6 Pt 2.1161.

15. Rapoport RM, Schwartz K, Murad F. Effect of sodiumpotassium pump inhibitors and membrane-depolarizing agents on sodium nitroprusside-induced relaxation and cyclic guanosine monophosphate accumulation in rat aorta. Circ Res 1985; 57: 164-170, doi: 10.1161/01.RES.57.1.164.

16. Harvey RD. Muscarinic receptor agonists and antagonists: effects on cardiovascular function. Handb Exp Pharmacol 2012; 208: 299-316, doi: 10.1007/978-3-642-23274-9.

17. Walch $L$, Brink $C$, Norel $X$. The muscarinic receptor subtypes in human blood vessels. Therapie 2001; 56: 223-226.

18. Cuthbert AW. Some effects of atropine on smooth muscle. Br J Pharmacol Chemother 1963; 21: 285-294, doi: 10.1111/ j.1476-5381.1963.tb01527.x.

19. Palacios M, Knowles RG, Palmer RM, Moncada S. Nitric oxide from L-arginine stimulates the soluble guanylate cyclase in adrenal glands. Biochem Biophys Res Commun 1989; 165: 802-809, doi: 10.1016/S0006-291X(89)80037-3.

20. Garthwaite J, Southam E, Boulton CL, Nielsen EB, Schmidt $\mathrm{K}$, Mayer $\mathrm{B}$. Potent and selective inhibition of nitric oxidesensitive guanylyl cyclase by $1 \mathrm{H}-(1,2,4)$ oxadiazolo(4,3-a) quinoxalin-1-one. Mol Pharmacol 1995; 48: 184-188.

21. Gadbois DM, Crissman HA, Tobey RA, Bradbury EM. Multiple kinase arrest points in the G1 phase of nontransformed mammalian cells are absent in transformed cells. Proc Natl Acad Sci U S A 1992; 89: 8626-8630, doi: 10.1073/ pnas.89.18.8626.

22. Bashkatova V, Kraus MM, Vanin A, Hornick A, Prast $H$. Comparative effects of NO-synthase inhibitor and NMDA antagonist on generation of nitric oxide and release of amino acids and acetylcholine in the rat brain elicited by amphetamine neurotoxicity. Ann N Y Acad Sci 2004; 1025: 221-230, doi: 10.1196/annals.1316.027.

23. Bashkatova V, Hornick A, Vanin A, Prast H. Antagonist of M1 muscarinic acetylcholine receptor prevents neurotoxicity induced by amphetamine via nitric oxide pathway. Ann N Y Acad Sci 2008; 1139: 172-176, doi: 10.1196/annals. 1432.004.

24. Schmid-Antomarchi $\mathrm{H}$, de Weille J, Fosset M, Lazdunski M. The antidiabetic sulfonylurea glibenclamide is a potent blocker of the ATP-modulated $\mathrm{K}+$ channel in insulin secreting cells. Biochem Biophys Res Commun 1987; 146: 21-25, doi: 10.1016/0006-291X(87)90684-X.

25. Ok SH, Han JY, Sung HJ, Yang SM, Park J, Kwon SC, et al. Ropivacaine-induced contraction is attenuated by both endothelial nitric oxide and voltage-dependent potassium channels in isolated rat aortae. Biomed Res Int 2013; 2013: 565271, doi: 10.1155/2013/565271.

26. Shieh CC, Kirsch GE. Mutational analysis of ion conduction and drug binding sites in the inner mouth of voltage-gated K+ channels. Biophys J 1994; 67: 2316-2325, doi: 10.1016/ S0006-3495(94)80718-0.

27. Mathie A, Wooltorton JR, Watkins CS. Voltage-activated potassium channels in mammalian neurons and their block by novel pharmacological agents. Gen Pharmacol 1998; 30: 13-24, doi: 10.1016/S0306-3623(97)00034-7.

28. Zografos $\mathrm{P}, \mathrm{Li} \mathrm{JH}, \mathrm{Kau} \mathrm{ST}$. Comparison of the in vitro effects of $\mathrm{K}^{+}$channel modulators on detrusor and portal vein strips from guinea pigs. Pharmacology 1992; 45: 216-230, doi: 10.1159/000139000. 
29. Pataricza J, Marton Z, Lengyel C, Toth M, Papp JG, Varro $A$, et al. Potassium channels sensitive to combination of charybdotoxin and apamin regulate the tone of diabetic isolated canine coronary arteries. Acta Physiol 2008; 194: 35-43, doi: 10.1111/j.1748-1716.2008.01859.x.

30. Qiu Y, Quilley J. Apamin/charybdotoxin-sensitive endothelial $\mathrm{K}^{+}$channels contribute to acetylcholine-induced, NO-dependent vasorelaxation of rat aorta. Med Sci Monit 2001; 7: $1129-1136$.

31. Lopez-Canales JS, Lozano-Cuenca J, Lopez-Canales OA, Aguilar-Carrasco JC, Aranda-Zepeda L, Lopez-Sanchez P, et al. Pharmacological characterization of mechanisms involved in the vasorelaxation produced by rosuvastatin in aortic rings from rats with a cafeteria-style diet. Clin Exp Pharmacol Physiol 2015; 42: 653-661, doi: 10.1111/1440-1681.12406.

32. Tottrup A, Kraglund K. Endothelium-dependent responses in small human mesenteric arteries. Physiol Res 2004; 53: 255-263.

33. Corriu C, Feletou M, Canet E, Vanhoutte PM. Endotheliumderived factors and hyperpolarization of the carotid artery of the guinea-pig. $\mathrm{Br} J$ Pharmacol 1996; 119: 959-964, doi: 10.1111/j.1476-5381.1996.tb15765.x.
34. Dusting GJ, Moncada S, Mullane KM, Vane JR. Implications of prostacyclin generation for modulation of vascular tone. Clin Sci Mol Med Suppl 1978; 4: 195s-198s.

35. Chen G, Suzuki H, Weston AH. Acetylcholine releases endothelium-derived hyperpolarizing factor and EDRF from rat blood vessels. Br J Pharmacol 1988; 95: 1165-1174, doi: 10.1111/j.1476-5381.1988.tb11752.x.

36. Hecker M, Bara AT, Bauersachs J, Busse R. Characterization of endothelium-derived hyperpolarizing factor as a cytochrome P450-derived arachidonic acid metabolite in mammals. J Physiol 1994; 481 (Part 2) 407-414.

37. Vane JR. Inhibition of prostaglandin synthesis as a mechanism of action for aspirin-like drugs. Nat New Biol 1971; 231: 232-235, doi: 10.1038/newbio231232a0.

38. Satav JG, Katyare SS, Fatterparker P, Sreenivasan A. Study of protein synthesis in rat liver mitochondria use of cycloheximide. Eur J Biochem 1977; 73: 287-296, doi: 10.1111/j.14321033.1977.tb11318.x.

39. Wu CC, Chen SJ, Yen MH. Different responses to acetylcholine in the presence of nitric oxide inhibitor in rat aortae and mesenteric arteries. Clin Exp Pharmacol Physiol 1993; 20: 405-412, doi: 10.1111/j.1440-1681.1993.tb01717.x. 